ANNALES

POLONICI MATHEMATICI

$\mathrm{XX}$ (1868)

\title{
On a biharmonic function in the unit disc
}

\author{
by Paulina Pycil (Poznań)
}

In this note we examine for a given function $f(t)$ with Fourier coefficients $a_{0}, a_{n}, b_{n}$ the biharmonic function $f(r, \varphi)$ defined in the disc $\left|r e^{i \varphi}\right|<1$ by

$$
f(r, \varphi)=\frac{a_{0}}{2}+\sum_{n=1}^{\infty}\left[1+\frac{n}{2}\left(1-r^{2}\right)\right] r^{n}\left(a_{n} \cos n \varphi+b_{n} \sin n \varphi\right) .
$$

It can easily be verified (cf. [3], pp. 395-400) that

$$
f(r, \varphi)=\frac{\left(1-r^{2}\right)^{2}}{2 \pi} \int_{-\pi}^{\pi} f(t) \frac{1-r \cos (t-\varphi)}{\left[1+r^{2}-2 r \cos (t-\varphi)\right]^{2}} d t
$$

for any $f(t)$ Lebesgue-integrable in $\langle-\pi, \pi\rangle$; these functions $f(t)$, with period $2 \pi$, are considered below. Our theorems complete the results announced in [2].

For the sake of brevity, we shall write

$$
K(r, t)=\frac{\left(1-r^{2}\right)^{2}(1-r \cos t)}{2 \pi\left[1+r^{2}-2 r \cos t\right]^{2}}=\frac{\left(1-r^{2}\right)^{2}\left(1-r+2 r \sin ^{2}(t / 2)\right)}{2 \pi\left[(1-r)^{2}+4 r \sin ^{2}(t / 2)\right]^{2}}
$$

Then, by (2),

$$
f(r, \varphi)=\int_{-\pi}^{\pi} f(t) K(r, t-\varphi) d t=\int_{0}^{\pi}[f(\varphi+t)+f(\varphi-t)] K(r, t) d t .
$$

In particular, the last representation together with (1) gives

$$
1=2 \int_{0}^{\pi} K(r, t) d t
$$

Hence

$$
f(r, \varphi)-f(\varphi)=\int_{0}^{\pi}[f(\varphi+t)-2 f(\varphi)+f(\varphi-t)] K(r, t) d t .
$$


The kernel $K(r, t)$ is positive decreasing in $t \epsilon\langle 0, \pi\rangle$ such that $K(r, \theta) \rightarrow 0$ as $r \rightarrow 1-$ for arbitrarily small $\theta>0$. Thus we have

$$
\lim _{r \rightarrow 1-} f(r, \varphi)=f(\varphi)
$$

at almost every $\varphi$, and the convergence is uniform over every closed interval of points of continuity.

Let $f(\varphi)$ be bounded in $\langle-\pi, \pi\rangle$ and let $\omega_{\varepsilon}(\delta)=\omega_{2}(\delta ; f)$ be the second modulus of smoothness of $f$, i.e.

$$
\omega_{\varepsilon}(\delta ; f)=\sup _{0 \leqslant t \leqslant \delta}\left\{\sup _{|\varphi| \leqslant \pi}|f(\varphi+t)-2 f(\varphi)+f(\varphi-t)|\right\} .
$$

We shall denote by $Z_{a}(0<\alpha \leqslant 2)$ the class of all these $f$ 's for which

$$
\omega_{2}(\delta ; f) \leqslant 2 \delta^{a} \quad \text { when } \quad 0 \leqslant \delta \leqslant \pi .
$$

Moreover, we shall write

$$
U_{\mathbf{a}}(r)=\sup _{f \in Z_{\boldsymbol{a}}}\left\{\max _{|\boldsymbol{q}| \leqslant \pi}|f(r, q)-f(\varphi)|\right\} .
$$

Theorem 1. The following asymptotic formula

$$
U_{u}(r)=\frac{2}{\pi} B\left(\frac{1+a}{2}, \frac{3-a}{2}\right)(1-r)^{a}+\left\{\begin{array}{llr}
O\left\{(1-r)^{9 a}\right\} & \text { for } & 0<a \\
O\left\{(1-r)^{1+a}\right\} & \text { for } & 1 / 2<a<1 / 2, \\
O\left\{(1-r)^{2}\right\} & \text { for } & 1<a<2
\end{array}\right.
$$

holds as $r \rightarrow 1-$.

Proof. By (3),

$$
\max _{|\varphi| \leqslant \pi}|f(r, \varphi)-f(\varphi)| \leqslant 2 \int_{i}^{\pi} t^{u} K(r, t) d t
$$

for any $f \in Z_{a}$. Therefore,

$$
\begin{aligned}
U_{a}(r) & \leqslant 2^{a+2} \int_{0}^{\pi / 2} t^{a} K(r, 2 t) d t \\
& =2^{a+2}\left[\int_{0}^{\pi / 2} \sin ^{a} t K(r, 2 t) d t+\int_{0}^{\pi / 2}\left(t^{a}-\sin ^{a} t\right) K(r, 2 t) d t\right]
\end{aligned}
$$

The function

$$
f_{a}(\varphi)=|2 \sin (\varphi / 2)|^{a} \quad(0<a \leqslant 2)
$$

is of class $Z_{a}$ (see [1]), and

$$
f_{a}(r, 0)-f_{a}(0)=2^{a+2} \int_{0}^{\pi / 2} \sin ^{a} t K(r, 2 t) d t
$$


$\left(f_{a}(r, \varphi)\right.$ is the function (1) for $\left.f_{a}(\varphi)\right)$; whence

$$
U_{a}(r) \geqslant 2^{a+2} \int_{0}^{\pi / 2} \sin ^{a} t K(r, 2 t) d t .
$$

Inequalities (4) and (5) lead to

$$
\begin{aligned}
U_{a}(r) & =2^{a+2}\left[\int_{0}^{\pi / 2} \sin ^{a} t K(r, 2 t) d t+C_{u}(r) \int_{0}^{\pi / 2}\left(t^{a}-\sin ^{a} t\right) K(r, 2 t) d t\right] \\
& =2^{a+\nu}\left[I_{1}+C_{a}(r) I_{2}\right], \quad \text { where } 0 \leqslant C_{a}(r) \leqslant 1 .
\end{aligned}
$$

Clearly,

$$
\begin{aligned}
I_{1} & =\int_{0}^{\pi / 4} \sin ^{u} t K(r, 2 t) d t+O\left\{(1-r)^{2}\right\} \\
& =\int_{0}^{\pi / 4} \sin ^{a} t \cos t K(r, 2 t) d t+\int_{0}^{\pi / 4} \sin ^{a} t(1-\cos t) K(r, 2 t) d t+O\left\{(1-r)^{2}\right\} \\
& =Y_{1}+Y_{2}+O\left\{(1-r)^{2}\right\} .
\end{aligned}
$$

A change of variable $4 r \sin ^{2} t /(1-r)^{2}=x$ gives

$$
\begin{aligned}
y_{1} & =\frac{\left(1-r^{2}\right)^{2}}{2 \pi} \int_{0}^{\pi / 4} \sin ^{a} t \cos t \frac{1-r+2 r \sin ^{2} t}{\left[(1-r)^{2}+4 r \sin ^{2} t\right]^{2}} d t \\
& =\frac{(1+r)^{2}(1-r)^{a}}{\pi \cdot 2^{a+4}(\sqrt{r})^{a+1}} \int_{0}^{(1-r)^{2}} x^{(a-1) / 2} \frac{2+(1-r) x}{(1+x)^{2}} d x \\
& =\frac{(1+r)^{2}(1-r)^{a}}{\pi \cdot 2^{a+3}(\sqrt{r})^{a+1}} \int_{0}^{\infty} \frac{x^{(a-1) / 2}}{(1+x)^{2}} d x+\left\{\begin{array}{lll}
O\left\{(1-r)^{1+a}\right\} & \text { for } & 0<a<1, \\
O\left\{(1-r)^{2}\right\} & \text { for } & 1<a<2 .
\end{array}\right.
\end{aligned}
$$

Since $(1-\cos t)=2 \sin ^{2}(t / 2) \leqslant 2 \sin t \cos t(0 \leqslant t \leqslant \pi / 4)$, we have

$$
\begin{aligned}
Y_{2} & \leqslant \frac{\left(1-r^{2}\right)^{2}}{\pi} \int_{0}^{\pi / 4} \sin ^{(1+a)} t \cos t \frac{1-r+2 r \sin ^{2} t}{\left[(1-r)^{2}+4 r \sin ^{2} t\right]^{2}} d t \\
& =\frac{(1+r)^{2}(1-r)^{1+a}}{\pi \cdot 2^{a+4}(\sqrt{r})^{a+2}} \int_{0}^{2 r /(1-r)^{2}} x^{a / 2} \frac{2+(1-r) x}{(1+x)^{2}} d x
\end{aligned}
$$

i.e.

$$
Y_{2}=\left\{\begin{array}{lll}
O\left\{(1-r)^{1+a}\right\} & \text { for } & 0<a<1, \\
O\left\{(1-r)^{2}\right\} & \text { for } & 1<\alpha<2 .
\end{array}\right.
$$


Thus we get

$$
I_{1}=\frac{(1+r)^{2}(1-r)^{a}}{\pi \cdot 2^{a+3}(\sqrt{r})^{a+1}} \int_{0}^{\infty} \frac{x^{(a-1) / 2}}{(1+x)^{2}} d x+\left\{\begin{array}{lll}
O\left\{(1-r)^{1+a}\right\} & \text { if } & 0<a<1, \\
O\left\{(1-r)^{2}\right\} & \text { if } & 1<a<2 .
\end{array}\right.
$$

Consider now the integral $I_{2}$. Suppose first that $0<a<1$. Then the inequality

$$
t^{a}-\sin ^{a} t \leqslant d_{\alpha} \sin ^{3 a} t \quad(0 \leqslant t \leqslant \pi / 2), \quad \text { where } \quad d_{a}=\frac{1}{(3 !)^{a}}\left(\frac{\pi}{2}\right)^{9 a},
$$

implies

$$
I_{2} \leqslant d_{a} \int_{0}^{\pi / 2} \sin ^{3 a} t K(r, 2 t) d t=d_{a} \int_{0}^{\pi / 4} \sin ^{3 a} t K(r, 2 t) d t+O\left\{(1-r)^{2}\right\} .
$$

Further,

$$
\begin{aligned}
I_{2} \leqslant & \sqrt{2} d_{a} \int_{0}^{\pi / 4} \sin ^{3 a} t \cos t K(r, 2 t) d t+O\left\{(1-r)^{2}\right\} \\
= & \frac{\sqrt{2} d_{a}(1+r)^{2}(1-r)^{3 a}}{\pi \cdot 2^{3 a+4}(V \cdot r)^{3 a+1}} \int_{0}^{2 r /(1-r)^{2}} x^{(3 a-1) / 2} \frac{2+(1-r) x}{(1+x)^{2}} d x+O\left\{(1-r)^{2}\right\} \\
= & \frac{\sqrt{2} d_{a}(1+r)^{2}(1-r)^{3 a}}{\pi \cdot 2^{3 a+a}(\sqrt{r})^{3 a+1}} \int_{0}^{\infty} \frac{x^{(3 a-1) / 2}}{(1+x)^{2}} d x+ \\
& +\left\{\begin{array}{llr}
O\left\{(1-r)^{3 a+1}\right\} & \text { for } \quad 0<a \leqslant 1 / 3 \\
O\left\{(1-r)^{2}\right\} & \text { for } \quad 1 / 3<a<1 .
\end{array}\right.
\end{aligned}
$$

Consequently,

$$
I_{2}=\left\{\begin{array}{llr}
O\left\{(1-r)^{9 a}\right\} & \text { for } & 0<a \leqslant 2 / 3 \\
O\left\{(1-r)^{2}\right\} & \text { for } & 2 / 3<a<1
\end{array}\right.
$$

In the case of $1<a<2$,

Hence

$$
t^{a}-\sin ^{\alpha} t \leqslant\left(\frac{t^{4}}{3}\right)^{a / 2} \leqslant \frac{1}{3^{a / 2}}\left(\frac{\pi}{2}\right)^{2 a} \sin ^{2 a} t \quad(0 \leqslant t \leqslant \pi / 2) .
$$

$$
\begin{aligned}
I_{2} & \leqslant \frac{1}{3^{a / 2}}\left(\frac{\pi}{2}\right)^{2 a} \frac{\left(1-r^{2}\right)^{2}}{2 \pi} \int_{0}^{\pi / 2} \sin ^{2} t \frac{1-r+2 r \sin ^{2} t}{\left[(1-r)^{2}+4 r \sin ^{2} t\right]^{2}} d t \\
& =\frac{1}{3^{a / 2}}\left(\frac{\pi}{2}\right)^{2 a} \frac{(2+r)}{16}(1-r)^{2},
\end{aligned}
$$

i.e.

$$
I_{2}=\left\{\begin{array}{llc}
O\left\{(1-r)^{3 a}\right\} & \text { for } & 0<a \leqslant 2 / 3, \\
O\left\{(1-r)^{2}\right\} & \text { for } \quad 2 / 3<a<1 \text { or } 1<a<2
\end{array}\right.
$$


Putting together the results, we obtain

$$
U_{\mathrm{a}}(r)=\frac{(1+r)^{2}(1-r)^{a}}{2 \pi(\bar{V} \bar{r})^{a+1}} \int_{0}^{\infty} \frac{x^{(\alpha-1) / 2}}{(1+x)^{2}} d x+\left\{\begin{array}{llr}
O\left\{(1-r)^{3 a}\right\} & \text { if } & 0<a \leqslant 1 / 2, \\
O\left\{(1-r)^{1+a}\right\} & \text { if } & 1 / 2<a<1, \\
O\left\{(1-r)^{2}\right\} & \text { if } & 1<a<2,
\end{array}\right.
$$

and the desired formula follows immediately.

Analogously, for $a=1$, the asymptotic relation

$$
U_{1}(r)=\frac{2}{\pi}(1-r)+O\left\{(1-r)^{2}|\ln (1-r)|\right\} \quad \text { as } \quad r \rightarrow 1-
$$

can also easily be deduced (cf. [2], theorem 3).

Notice also that the inequalities

$$
I(r) \leqslant U_{2}(r) \leqslant(\pi / 2)^{2} I(r),
$$

where $I(r)=2^{4} \int_{0}^{\pi / 2} \sin ^{2} t K(r, 2 t) d t=(2+r)(1-r)^{2}$, imply

$$
2 \leqslant U_{2}(r) /(1-r)^{2} \leqslant 3(\pi / 2)^{2} .
$$

ThEorem 2. Suppose that for certain fixed $\varphi_{0}, a(0<a<2)$ the finite limit

$$
\lim _{t \rightarrow 0+} \frac{f\left(\varphi_{0}+t\right)-2 f\left(\varphi_{0}\right)+f\left(\varphi_{0}-t\right)}{t^{a}}=l
$$

exists, and that $f\left(\varphi_{0}+t\right)+f\left(\varphi_{0}-t\right)$ remains bounded in $\langle 0, \pi\rangle$. Then

$$
f\left(r, \varphi_{0}\right)=f\left(\varphi_{0}\right)+\frac{l}{\pi} B\left(\frac{1+a}{2}, \frac{3-a}{2}\right)(1-r)^{a}+o\left\{(1-r)^{a}\right\} \quad \text { as } \quad r \rightarrow 1-.
$$

Proof. By hypothesis, we can write

$$
f\left(\varphi_{0}+t\right)-2 f\left(\varphi_{0}\right)+f\left(\varphi_{0}-t\right)=[l+\lambda(t)](2 \sin (t / 2))^{a},
$$

where the function $\lambda(t)$ is bounded in $(0, \pi\rangle$, say $|\lambda(t)| \leqslant M$, and $\lambda(t) \rightarrow 0$ as $t \rightarrow 0+$.

Applying (3) and (6),

$$
\begin{aligned}
f\left(r, \varphi_{0}\right)-f\left(\varphi_{0}\right) & =l \int_{0}^{\pi}(2 \sin (t / 2))^{a} K(r, t) d t+\int_{0}^{\pi}(2 \sin (t / 2))^{a} \lambda(t) K(r, t) d t \\
& =l I_{a}(r)+Y_{a}(r) .
\end{aligned}
$$

Given $\varepsilon>0$, there is a $\delta>0$ such that $|\lambda(t)|<\varepsilon$ for $0<t \leqslant \delta$. Then

$$
\begin{aligned}
\left|\frac{Y_{a}(r)}{I_{a}(r)}\right| & \leqslant \frac{1}{I_{a}(r)}\left[\varepsilon \int_{0}^{\delta}\left(2 \sin \frac{t}{2}\right)^{a} K(r, t) d t+M \int_{\delta}^{\pi}\left(2 \sin \frac{t}{2}\right)^{a} K(r, t) d t\right] \\
& \leqslant \varepsilon+\frac{M}{I_{a}(r)} \int_{0}^{\pi}\left(2 \sin \frac{t}{2}\right)^{a} K(r, t) d t .
\end{aligned}
$$


The last integral is $O\left\{(1-r)^{2}\right\}$. Further,

$$
\begin{gathered}
I_{a}(r)=2^{a+1} \int_{0}^{\pi / 2} \sin ^{a} t K(r, 2 t) d t \\
=\frac{1}{\pi} B\left(\frac{1+a}{2}, \frac{3-a}{2}\right)(1-r)^{a}+\left\{\begin{array}{lll}
O\left\{(1-r)^{1+a}\right\} & \text { if } & 0<a<1, \\
O\left\{(1-r)^{2}\right\} & \text { if } & 1<a<2, \\
I_{1}(r)=\frac{1}{\pi}(1-r)+O\left\{(1-r)^{2}|\ln (1-r)|\right\} .
\end{array}\right.
\end{gathered}
$$

Consequently

$$
I_{a}(r)=\frac{1}{\pi} B\left(\frac{1+a}{2}, \frac{3-a}{2}\right)(1-r)^{a}+o\left\{(1-r)^{a}\right\} \quad \text { for } \quad 0<a<2 .
$$

Thus we have $Y_{a}(r)=O\left\{I_{a}(r)\right\}$ as $r \rightarrow 1-$, and the proof is completed.

THEOREM 3. Let $f(\varphi)$ be bounded in $\langle-\pi, \pi\rangle$ and let $\omega_{2}(\delta)$ signify the second modulus of smoothness of this function. Then

$$
\max _{|\varphi| \leqslant \pi}\left|\frac{\partial^{2} f(r, \varphi)}{\partial \varphi^{2}}\right| \leqslant C \frac{r \omega_{2}(1-r)}{(1-r)^{2}} \quad \text { for } \quad 0 \leqslant r<1,
$$

with an absolute constant $C$.

Proof. Starting with the identity

$$
\frac{\partial^{2} f(r, \varphi)}{\partial \varphi^{2}}=\int_{-\pi}^{\pi} f(\varphi+t) \frac{\partial^{\alpha} K(r, t)}{\partial t^{2}} d t
$$

and observing that, by (1), (2), $\partial^{2} K(r, t) / \partial t^{2}$ is even and its integral extended over the interval $\langle-\pi, \pi\rangle$ vanishes, we obtain

$$
\frac{\partial^{2} f(r, \varphi)}{\partial \varphi^{2}}=\int_{0}^{\pi}[f(\varphi+t)-2 f(\varphi)+f(\varphi-t)] \frac{\partial^{2} K(r, t)}{\partial t^{2}} d t .
$$

Direct computation gives

$$
\frac{\partial^{2} K(r, t)}{\partial t^{2}}=\frac{r\left(1-r^{2}\right)^{2}}{2 \pi}\left[\frac{2 r \cos 2 t-\left(3-r^{2}\right) \cos t}{\left(1+r^{2}-2 r \cos t\right)^{2}}+\frac{6 r \sin ^{2} t\left(3-r^{2}-2 r \cos t\right)}{\left(1+r^{2}-2 r \cos t\right)^{4}}\right] .
$$

Hence

where

$$
\left|\frac{\partial^{2} f(r, \varphi)}{\partial \varphi^{2}}\right| \leqslant \frac{r\left(1-r^{2}\right)^{2}}{2 \pi} \int_{0}^{\pi} \omega_{2}(t)\left[Q_{1}(r, t)+Q_{2}(r, t)\right] d t
$$

$$
\begin{aligned}
& Q_{1}(r, t)=\frac{2(1-r)(3+r)+t^{2}\left(3+8 r-r^{2}\right)}{2\left[(1-r)^{2}+4 r t^{2} / \pi^{2}\right]^{3}}, \\
& Q_{2}(r, t)=\frac{6 r t^{2}\left[(1-r)(3+r)+r t^{2}\right]}{\left[(1-r)^{2}+4 r t^{2} / \pi^{2}\right]^{4}}
\end{aligned}
$$


Now, it is easily seen that if $0 \leqslant r \leqslant 1 / 2$,

$$
\left|\frac{\partial^{2} f(r, \varphi)}{\partial \varphi^{2}}\right| \leqslant \frac{16 r}{\pi} \int_{0}^{\pi} \omega_{2}(t)\left(7+91 t^{2}+12 t^{4}\right) d t
$$

and by the inequality

we have

$$
\omega_{2}(t) \leqslant\left\{(1-r+t)^{2} \omega_{2}(1-r)\right\} /(1-r)^{2},
$$

$$
\max _{|\varphi| \leqslant \pi}\left|\frac{\partial^{2} f(r, \varphi)}{\partial \varphi^{2}}\right| \leqslant C_{1} \frac{r \omega_{2}(1-r)}{(1-r)^{2}} \quad\left(C_{1}=\text { const }\right) .
$$

In the case of $1 / 2<r<1$,

$$
\left|\frac{\partial^{2} f(r, \varphi)}{\partial \varphi^{2}}\right| \leqslant \frac{r(1+r)^{2}(1-r)^{3}}{4 \sqrt{r}} \int_{0}^{2 \sqrt{r /(1-r)}} \omega_{2}\left(\frac{\pi(1-r)}{2 \sqrt{r}} x\right)\left[Q_{1}^{*}(r, x)+Q_{2}^{*}(r, x)\right] d x,
$$

where $Q_{v}^{*}(r, x)=Q,(r, \pi(1-r) x / 2 \downarrow \bar{r})$. Applying the estimate

we get

$$
\omega_{2}\left(\frac{\pi(1-r)}{2 \mathrm{~J}^{\prime}} x\right) \leqslant\left(1+\frac{\pi}{2 \mathrm{~J}^{\prime} r} x\right)^{2} \omega_{2}(1-r)
$$

$$
\left|\frac{\hat{\partial}^{2} f(r, \varphi)}{c \varphi^{2}}\right| \leqslant \frac{r\left(\omega_{2}(1-r)\right.}{2(1-r)^{2}} \int_{0}^{\infty}\left(1+\frac{\pi}{2 \sqrt{r}} x\right)^{2}\left[\frac{32+6 \pi^{2} x^{2}}{\left(1+x^{2}\right)^{3}}+\frac{3 \pi^{2} x^{2}\left(32+\pi^{2} x^{2}\right)}{2\left(1+x^{2}\right)^{4}}\right] d x .
$$

Consequently

$$
\max _{|\varphi| \leqslant \pi}\left|\frac{\partial^{2} f(r, \varphi)}{\partial \varphi^{2}}\right| \leqslant C_{2} \frac{r \omega_{2}(1-r)}{(1-r)^{2}} \quad\left(C_{2}=\text { const }\right) .
$$

The proof is thus completed.

THeorem 4. If a finite derivative $f^{(s)}\left(\varphi_{0}\right)(s$ is 1 or 2$)$ exists, then

$$
\lim _{(r, \varphi)} \frac{\partial^{8} f(r, \varphi)}{\partial \varphi^{8}}=f^{(s)}\left(\varphi_{0}\right)
$$

as $(r, \varphi)$ approaches $\left(1, \varphi_{0}\right)$ in such a way that $\left(\varphi-\varphi_{0}\right) /(1-r)$ remains bounded (cf. [4], pp. 100-101).

Proof. Let us confine ourselves to the case of $-\pi<\varphi_{0}<\pi, s=2$. The function

$$
f_{*}(t)=f\left(\varphi_{0}\right)+f^{\prime}\left(\varphi_{0}\right) \sin \left(t-\varphi_{0}\right)+\frac{1}{2} f^{\prime \prime}\left(\varphi_{0}\right) \sin ^{2}\left(t-\varphi_{0}\right)
$$

is $2 \pi$-periodic infinite-differentiable in $(-\infty, \infty)$,

$$
f_{*}^{(p)}\left(\varphi_{0}\right)=f^{(p)}\left(\varphi_{0}\right) \quad \text { for } \quad v=0,1,2 \text {. }
$$


Moreover,

$$
\eta(t)=\left(f(t)-f_{*}(t)\right) / \sin ^{2}\left(t-\varphi_{0}\right) \quad \text { tends to zero as } t \rightarrow \varphi_{0} .
$$

As for the Poisson integral,

$$
\frac{\partial^{2} f_{*}(r, \varphi)}{\partial \varphi^{2}}=\int_{-\pi}^{\pi} f_{*}(t) \frac{\partial^{2} K(r, t-\varphi)}{\partial t^{2}} d t=\int_{-\pi}^{\pi} f_{*}^{\prime \prime}(t) K(r, t-\varphi) d t
$$

and

$$
\lim _{\substack{r \rightarrow 1-\\ \varphi \rightarrow \varphi_{0}}} \int_{-\pi}^{\pi} f_{*}^{\prime \prime}(t) K(r, t-\varphi) d t=f_{*}^{\prime \prime}\left(\varphi_{0}\right)=f^{\prime \prime}\left(\varphi_{0}\right)
$$

Writing $h(t)=f(t)-f_{*}(t)$, we have

and

$$
\frac{\partial^{2} f(r, \varphi)}{\partial \varphi^{2}}=\frac{\partial^{2} f_{*}(r, \varphi)}{\partial \varphi^{2}}+\frac{\partial^{2} h(r, \varphi)}{\partial \varphi^{2}},
$$

$$
\frac{\partial^{2} h(r, \varphi)}{\partial \varphi^{2}}=\int_{-\pi}^{\pi} \eta(t) \sin ^{2}\left(t-\varphi_{0}\right) \frac{\partial^{2} K(r, t-\varphi)}{\partial t^{2}} d t
$$

Given any $\varepsilon>0$, let $\delta\left(0<\delta \leqslant \min \left(\pi-\varphi_{0}, \pi+\varphi_{0}\right)\right)$ be such that $|\eta(t)| \leqslant \varepsilon$ for $\left|t-\varphi_{0}\right| \leqslant \delta$. Then,

$$
\left|\frac{\partial^{2} h(r, \varphi)}{\partial \varphi^{2}}\right| \leqslant \varepsilon \int_{\left|\ell-\varphi_{0}\right| \leqslant \delta} \sin ^{2}\left(t--\varphi_{0}\right)\left|\frac{\partial^{2} K(r, t-\varphi)}{\partial t^{2}}\right| d t+\left|I_{\delta}(r, \varphi)\right|,
$$

where

$$
I_{\delta}(r, \varphi)=\int_{\left|t-\varphi_{0}\right|>\delta} \eta(t) \sin ^{2}\left(t-\varphi_{0}\right) \frac{\partial^{2} K(r, t-\varphi)}{\partial t^{2}} d t .
$$

Clearly,

$$
\begin{aligned}
& \int_{\left|t-\varphi_{0}\right| \leqslant \delta} \sin ^{2}\left(t-\varphi_{0}\right)\left|\frac{\partial^{2} K(r, t-\varphi)}{\partial t^{2}}\right| d t \\
& \leqslant \int_{-\pi}^{\pi} \sin ^{2}\left(t-\varphi_{0}\right)\left|\frac{\partial^{2} K(r, t-\varphi)}{\partial t^{2}}\right| d t \\
&= \int_{-\pi}^{\pi} \sin ^{2} t\left|\frac{\partial^{2} K(r, t)}{\partial t^{2}}\right| d t+\int_{-\pi}^{\pi}\left[\sin ^{2}\left(t+\varphi-\varphi_{0}\right)-\sin ^{2} t\right] \times \\
& \times\left|\frac{\partial^{2} K(r, t)}{\partial t^{2}}\right| d t=A+B .
\end{aligned}
$$


The integral $A$ is uniformly bounded in $r(0 \leqslant r<1)$,

$$
\begin{aligned}
|B| & \leqslant\left|\varphi-\varphi_{0}\right| \int_{-\pi}^{\pi}\left|\sin \left(t+\varphi-\varphi_{0}\right)+\sin t\right|\left|\frac{\partial^{2} K(r, t)}{\partial t^{2}}\right| d t \\
& \leqslant 2\left|\varphi-\varphi_{0}\right|\left\{2 \int_{0}^{\pi} \sin t\left|\frac{\partial^{2} K(r, t)}{\partial t^{2}}\right| d t+\left|\varphi-\varphi_{0}\right| \int_{0}^{\pi}\left|\frac{\partial^{2} K(r, t)}{\partial t^{2}}\right| d t\right\} \\
& \leqslant M_{1}\left|\varphi-\varphi_{0}\right| /(1-r)+M_{2}\left(\varphi-\varphi_{0}\right)^{2} /(1-r)^{2} \quad(M,=\text { const }) .
\end{aligned}
$$

Also, it can easily be observed that

$$
\lim I_{\delta}(r, \varphi)=0 \quad \text { as } \quad(r, \varphi) \rightarrow\left(1, \varphi_{0}\right) .
$$

Putting together the results, we get the above-mentioned assertion.

ThEOREM 5. For any bounded function $f(\varphi)$ with modulus $\omega_{2}(\delta)$, we have

$$
\max _{|\varphi| \leqslant \pi}\left|\frac{\partial f(r, \varphi)}{\partial r}\right| \leqslant M \frac{\omega_{2}(1-r)}{1-r} \quad \text { for } \quad 0<r_{0} \leqslant r<1,
$$

where $M$ is a constant depending only on $r_{0}$. In particular, if $\omega_{2}(\delta)=o(\delta)$, the relation

$$
\lim _{r \rightarrow 1-} \frac{\partial f(r, \varphi)}{\partial r}=0
$$

holds uniformly in $\varphi$.

Proof. Let $A_{0}=a_{0} / 2, A_{n}(\varphi)=a_{n} \cos n \varphi+b_{n} \sin n \varphi$, where $a_{0}, a_{n}, b_{n}$ are the Fourier coefficients of $f$. In view of (1),

$$
\frac{\partial f(r, \varphi)}{\partial r}=\left(1-r^{2}\right) \sum_{n=1}^{\infty}\left(n+\frac{n^{2}}{2}\right) r^{n-1} A_{n}(\varphi) .
$$

Denote by $f^{*}(r, \varphi)$ the Abel-Poisson means of the Fourier series of $f$, i.e.

$$
f^{*}(r, \varphi)=A_{0}+\sum_{n=1}^{\infty} r^{n} A_{n}(\varphi)
$$

and write

Then, by (1),

$$
S_{r}(\varphi)=\sum_{n=1}^{\infty} n r^{n} A_{n}(\varphi), \quad T_{r}(\varphi)=\sum_{n=1}^{\infty} n^{2} r^{n} A_{n}(\varphi) .
$$

$$
f(r, \varphi)=f^{*}(r, \varphi)+\frac{1}{2}\left(1-r^{2}\right) S_{r}(\varphi) .
$$

Applying Theorem 1 of [2], and observing that

$$
\left|f^{*}(r, \varphi)-f(\varphi)\right| \leqslant C_{3} \frac{\omega_{2}(1-r)}{1-r} \quad \text { for } \quad 0 \leqslant r<1 \text {, }
$$


we get

$$
\left|f(r, \varphi)-f^{*}(r, \varphi)\right| \leqslant C_{4} \frac{\omega_{2}(1-r)}{1-r} \quad\left(C_{3,4}=\text { const }\right)
$$

whence

$$
\left(1-r^{2}\right)\left|S_{r}(\varphi)\right| \leqslant 2 C_{4} \frac{\omega_{2}(1-r)}{1-r} \quad \text { for } \quad 0 \leqslant r<1 .
$$

It is easily seen (cf. [4], pp. 108-109) that if $0 \leqslant r<1$,

Thus

$$
\left|T_{r}(\varphi)\right|=\left|\frac{\partial^{2} f^{*}(r, \varphi)}{\partial \varphi^{2}}\right| \leqslant C_{5} \frac{r \omega_{2}(1-r)}{(1-r)^{2}} \quad\left(C_{5}=\text { const }\right) .
$$

$$
\begin{aligned}
\left|\frac{\partial f(r, \varphi)}{\partial r}\right| & =\frac{1-r^{2}}{r}\left|S_{r}(\varphi)+\frac{1}{2} T_{r}(\varphi)\right| \\
& \leqslant\left(\frac{2 C_{4}}{r}+\frac{(1+r) C_{5}}{2}\right) \frac{\omega_{2}(1-r)}{1-r} \leqslant M \frac{\omega_{2}(1-r)}{1-r} \quad \text { as } \quad r \geqslant r_{0} .
\end{aligned}
$$

For the whole class $Z_{a}$ the estimate in the last theorem cannot be improved. Indeed, the function

$$
f(\varphi)=K_{a} \sum_{n=1}^{\infty} \frac{\cos n \varphi}{n^{1+a}} \quad(0<a<2),
$$

with a suitable constant $K_{a}$, is of class $Z_{a}$. Its Abel-Poisson means possesses the derivative

Since

$$
\frac{\partial^{2} f^{*}(r, \varphi)}{\partial \varphi^{2}}=-K_{a} \sum_{n=1}^{\infty} n^{1-a r^{n}} \cos n \varphi \quad(0 \leqslant r<1) .
$$

where

$$
\sum_{n=1}^{\infty} n^{1-a} r^{n}=\Gamma(\alpha) /(1-r)^{2-a}+\varrho r(\alpha),
$$

$$
\varrho_{r}(a)=\left\{\begin{array}{lll}
O\left\{(1-r)^{a-1}\right\} & \text { for } & 0<a<1, \\
O\{|\ln (1-r)|\} & \text { for } & a=1, \\
O(1) & \text { for } & 1<a<2
\end{array}\right.
$$

as $r \rightarrow 1-$ (see [4], pp. 76-77), we have

$$
\left|\frac{\partial^{2} f^{*}(r, \varphi)}{\partial \varphi^{2}}\right|_{\varphi=0} \mid \geqslant \frac{C_{0}}{(1-r)^{2-a}} \quad \text { for } \quad 0<r_{1} \leqslant r<1,
$$

where $C_{a}$ is a positive constant depending only on $\alpha$ and $r_{1}$. Now, it can easily be observed that there is a positive constant $C_{7}$ such that

$$
\max _{|\varphi| \leqslant \pi}\left|\frac{\partial f(r, \varphi)}{\partial r}\right| \geqslant \frac{C_{7}}{(1-r)^{1-a}} \quad \text { for } \quad 0<r_{1} \leqslant r<1 \text {. }
$$


The biharmonic function generated by

$$
f(\varphi)=4 \sin ^{2}(\varphi / 2)=2(1-\cos \varphi)
$$

belonging to $Z_{2}$ is of the form:

Hence,

$$
f(r, \varphi)=2-\left(3-r^{2}\right) r \cos \varphi
$$

$$
\begin{gathered}
\max _{|\varphi| \leqslant \pi}\left|\frac{\partial f(r, \varphi)}{\partial r}\right|=3\left(1-r^{2}\right) \geqslant 3(1-r) \quad \text { for } \quad 0 \leqslant r<1 . \\
\text { If } f(\varphi)=|2 \sin (\varphi / 2)|, \\
\lim _{r \rightarrow 1-} \frac{\partial f(r, 0)}{\partial r}=-\frac{2}{\pi}<0 .
\end{gathered}
$$

Thus, there exist functions $f(\varphi)$ satisfying the Lipschitz condition for which relation (7) is false at some $\varphi$.

Evidently, if $f(\varphi)$ possesses a finite second derivative in a set $E$, the relation

$$
\lim _{r \rightarrow 1-} \frac{\partial^{2} f^{*}(r, \varphi)}{\partial \varphi^{2}}=f^{\prime \prime}(\varphi)
$$

implies (7) for any $\varphi \in E$.

\section{Relerences}

[1] Л. И. Баусов, О приближсении функиий класса $Z_{a}$ положсительными методами суммирования рядов Фурье, Успехи Мат. Наук, 16 (1961), 3 (99), pp. 143-149.

[2] С. Каниев, Об уклонении бигармонических в круге функиий от их граничных значений, Доклады Акад. Наук СССР, 153 (1963), pp. 995-998.

[3] А. Н. Тихонов и А. А. Самарский, Уравнения математической физики, Москва-Ленинград 1951.

[4] A. Zygmund, Trigonometric series, I, Cambridge 1959.

KATEDRA MATEMATYKI II, UNIWERSYTET IM. A. MICKIEWICZA, POZNAN DEPARTMENT OF MATHEMATICS II, A. MICKIEWICZ UNIVERSITY, POZNAN 